\title{
Metastatic adenocarcinoma of bilateral cavernous sinus and optic nerve with unknown primary mimicking orbital pseudotumor
}

\author{
Rajiv Kumar, C. E. Deopujari, Rajan Shah, Ashish Kumar \\ Department of Neurosurgery, Bombay Hospital Institute of Medical Sciences, 12 New Marine lines, Mumbai - 400 020, India
}

\author{
Address for correspondence: \\ Dr. Rajiv Kumar, \\ Room No. 126B, MRC building, \\ First floor, Bombay Hospital, \\ 12 New Marine lines, \\ Mumbai - 400 020, India. \\ E-mail: rajivsahumch@yahoo.co.in
}

DOI: $10.4103 / 0028-3886.48807$

\begin{abstract}
Abstrat
Bilateral cavernous sinus and optic nerve metastases is extremely rare. We report an extremely rare case of metastatic adenocarcinoma of bilateral cavernous sinus and optic nerve with unknown primary presenting as orbital pseudotumor. She underwent bilateral optic nerve decompression and the diagnosis was established by biopsy.
\end{abstract}

Key words: Bilateral cavernous sinus metastasis, metastatic adenocarcinoma, orbital psuedotumor

\section{Introduction}

Metastases to the cavernous sinus are uncommon, even though the incidence of brain metastases in patients with systemic cancer is 30 to $40 \%$. Cavernous sinus metastases commonly presents with headache, periorbital pain, progressive ophthalmoplegia and unilateral facial numbness. ${ }^{[1-7]}$ Rarely, they can have unusual presentation. We report a rare case of metastatic adenocarcinoma of bilateral cavernous sinus and optic nerve with unknown primary presenting as orbital pseudotumor.

\section{Case Report}

A 58-year-old female, non-diabetic, presented one year back with complaints of right-sided headache, and right forehead and facial hypoesthesia of one month duration. Examination at that time showed $80 \%$ hypoesthesia in right V1 and 20-40\% hypoesthesia in right V2 and V3 dermatomes. There was mild bilateral proptosis with full range ocular movements. Ocular fundi and other systems were normal. Magnetic resonance imaging (MRI) of brain showed soft tissue proliferation in the right cavernous sinus with extension into the orbital apex with mild contrast enhancement and normal ventricles. Erythrocyte sedimentation rate at first hour was $42 \mathrm{~mm}$.
She was started on steroid. After six weeks of steroid, her headache and hypoesthesia marginally improved. Repeat MRI of brain at three months follow-up showed almost the same findings. Two months later she had recurrence of symptoms, which were treated with another course of steroids for six weeks. MRI brain done two months later showed in addition to the right-side lesion, thickening of the left cavernous sinus. In the meantime her headache resolved. After five months, she presented with rapidly progressive visual deterioration of left eye associated with headache and facial hypoesthesia. On examination she had visual acuity of $6 / 24$ in the right eye, while there was no PL/PR in the left eye. She had bilateral proptosis, right third and sixth cranial nerve palsies with decreased sensation of bilateral V1 and V2 dermatomes. Ocular fundi were normal. Repeat MRI of brain showed contrast-enhancing bilateral cavernous sinus lesion extending through the superior orbital fissure into the orbital apex, and inferiorly extending up to the foramen ovale [Figure 1, Figure 2]. In view of the rapidly progressive visual deterioration, no response to steroid, and no definitive diagnosis it was decided to do bilateral optic nerve decompression and biopsy of the lesion. She underwent bifrontal craniotomy and bilateral optic nerve decompression. Both the optic nerves were surrounded by inflamed tissue which was biopsied. The pathological diagnosis was metastatic adenocarcinoma 
[Figure 3, Figure 4]. Work-up for primary; CT scan chest, neck, and paranasal sinuses, ultra sound of abdomen and pelvis, mammography Pap smear, bone scan, and tumor markers were negative. She was advised whole brain radiotherapy (WBRT) followed by chemotherapy.

\section{Discussion}

The intracranial compartment is a common site for metastases. Common sites are brain parenchyma, leptomeninges, dura and skull. Common primary sites metastasizing to the cavernous sinus are breast, prostate, lungs, renal, uterus, testis, melanoma, liver, stomach, squamous cell carcinoma from various head and neck sites, parotid, thyroid, and lymphoma. Metastatic tumor involving cavernous sinus and optic nerve are rare, even rarer is its bilateral involvement with an unknown primary. On reviewing the literature we found only few cases,. ${ }^{[1-4]}$ Metastatic tumors to the cavernous sinus accounted for $23 \%$ of the 102 parasellar lesions reviewed

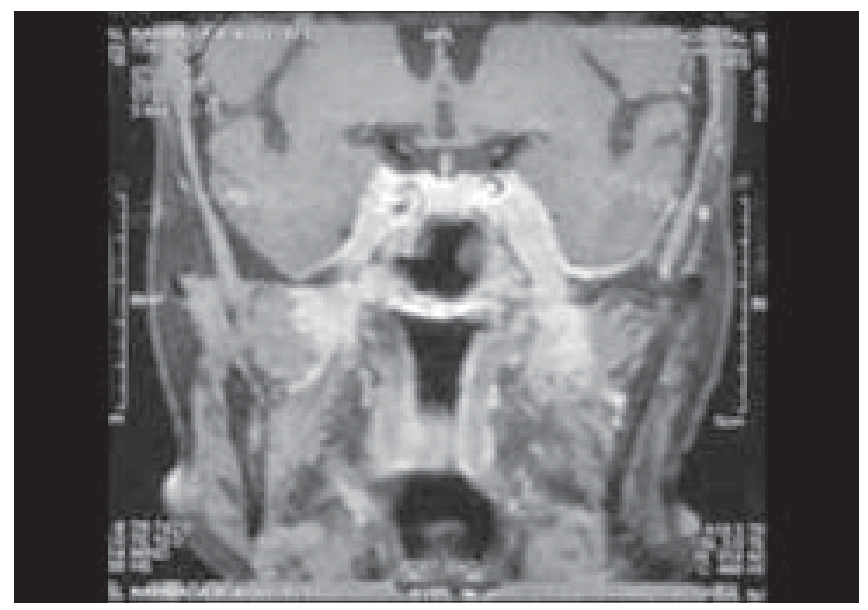

Figure 1: Coronal postcontrast MRI showing contrast-enhancing lesion involving both cavernous sinuses extending into the orbital apex

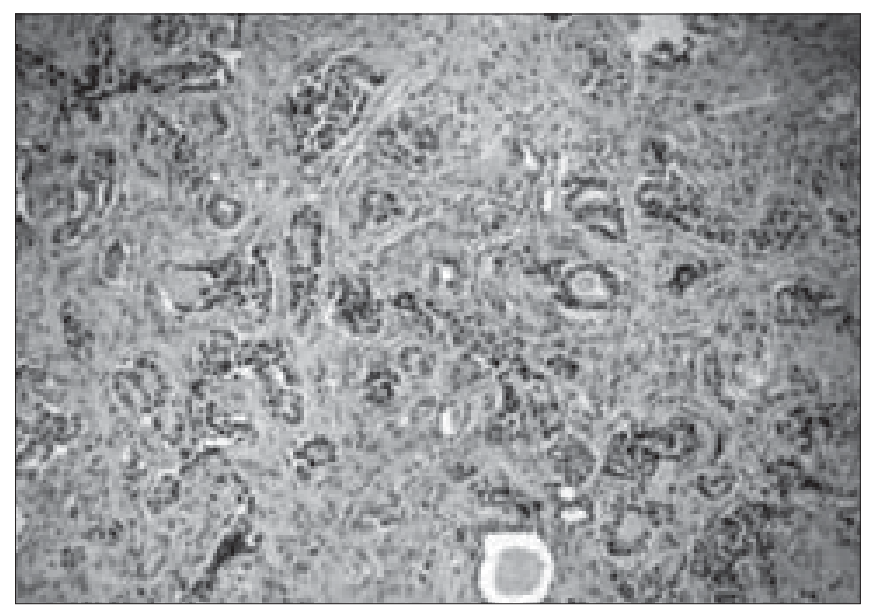

Figure 3: Photomicrograph (H\&E x100) showing haphazardly arranged tubuloglandular cells with mucin contents. Also seen is the mitotic activity with hypochromatic nuclei by Thomas and Yoss. ${ }^{\left[{ }^{[5]}\right.}$ A malignant lesion mostly invades the cavernous sinus by direct extension from adjacent compartments, ${ }^{[6]}$ such as, nasopharynx, infratemporal fossa, orbit and paranasal sinuses. Perineural invasion of cavernous sinus also occurs rarely and has been seen with adenoid cystic carcinoma. Cavernous sinus tumors most commonly present as cavernous sinus syndrome. Acute or slowly progressive ophthalmoplegia with diplopia is the dominant presentation. Symptoms of metastases to the cavernous sinus often include persistent painful dysesthesia in trigeminal distribution. Intrinsic bilateral cavernous sinus involvement with tumor usually produces early loss of trigeminal sensation. Most such cases in the literature have shown orbital or optic nerve involvement. ${ }^{[1]}$ Nakano et al reviewed 17 cases of unilateral cavernous sinus tumor due to distant metastases and found that all the cases had external ophthalmoplegia. Trigeminal nerve palsy was present in 13 cases. The optic nerve was involved in four cases. All the four cases had total ophthalmoplegia. ${ }^{[7]}$

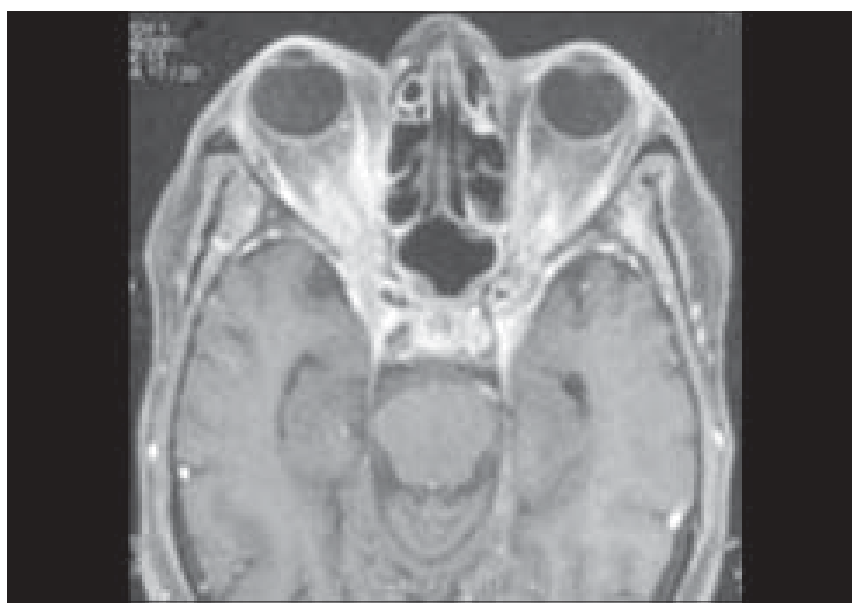

Figure 2: Axial postcontrast MRI showing contrast-enhancing lesion involving both cavernous sinuses extending into the orbital apex



Figure 4: Photomicrograph (H\&E x200) showing haphazardly arranged tubuloglandular cells with mucin contents. Also seen is the mitotic activity with hypochromatic nuclei 
In the present case, patient initially presented with insidious onset of symptoms and signs with imaging, suggestive of orbital pseudotumor. She also responded partially to steroids. Her symptoms kept waxing and waning for almost a year. The interesting finding in this case was the prolonged duration of symptoms with almost no changes on imaging, until patient presented with deterioration in her vision. For a metastatic lesion to remain unchanged for such a long period is quite unusual. The role of biopsy in patients with known systemic cancer with MRI-confirmed cavernous sinus lesions, is unclear. ${ }^{[8]}$ There is no clear indication for surgical resection of a cavernous sinus metastases. ${ }^{[8]}$ Total resection of these lesions is challenging and attempted only when the symptoms are disabling. WBRT remains the treatment of choice and provides effective symptom relief in the majority of patients. ${ }^{[9]}$ Even with WBRT median survival remains unaltered at approximately four to six months. ${ }^{[10]}$ Gamma knife radiosurgery could be a option for small benign or residual tumors involving the cavernous sinus. It is also very useful as part of comprehensive therapy for metastatic tumors in the cavernous sinus. ${ }^{[11]}$ Whenever malignant tumors involve the cavernous sinus, cure is infrequently possible; therefore, the aim of all treatment is to provide palliation of symptoms and good quality of life. ${ }^{[8]}$

\section{References}

1. Mills RP, Insalaco SJ, Joseph A. Bilateral cavernous sinus metastasis and ophthalmoplegia: Case report. J Neurosurg 1981;55:463-6.

2. Post MJ, Mendez DR, Kline LB, Acker JD, Glaser JS. Metastatic disease to the cavernous sinus: Clinical syndrome and CT diagnosis. J Comput Assist Tomogr 1985;9:115-20.

3. Yildirim N, Oksüzoğlu B, Vural M, Han O, Zengin N. Case report: Cavernous sinus metastasis of the parotid carcinoma: A very unusual case. J Neurooncol 2005;73:181-3.

4. Takami T, Ohata K, Tsuyuguchi N, Mao Y, Inoue Y, Wakasa K, et al. Cavernous sinus metastasis from thyroid papillary adenocarcinoma. J Clin Neurosei 2002;9:598-600.

5. Thomas JE, Yoss RE. The parasellar syndrome: Problems in determining etiology. Mayo Clin Proc 1970;45:617-23.

6. Krisht Ali F, Kadri Paulo AS, Raja Ali. Pathology of the cavernous sinus. Tech Neurosurg 2003;8:204-10.

7. Nakano N, Sato M, Miyasaka S, Yoshihara M, Ishikawa H. Clinical features of seventeen cases of cavernous sinus tumour due to distant metastasis. Jpn J Clin Ophthalmol 2000;54:599-602.

8. Panigrahi M, Arun P. Uncommon cavernous sinus lesions: A review. Progr Clin Neurosci 2007;22:237-47.

9. Vikram B, Chu FC. Radiation therapy for metastases to the base of the skull. Radiology 1979;130:465-8.

10. Minesh PM, Deepak K. Current strategies in whole brain radiation therapy for brain metastasis. Neurosurgery (suppl) 2005;57:33-44.

11. Liu AL, Wang C, Sun S, Wang M, Liu P. Gamma knife radiosurgery for tumors involving the cavernous sinus: Stereotact Funct Neurosurg 2005;83:45-51.

Accepted on 18-01-2009

Source of Support: Nil, Conflict of Interest: None declared. 